\title{
EFEITO DO METILFENIDATO NO PROCESSAMENTO AUDITIVO EM CRIANÇAS E ADOLESCENTES COM TRANSTORNO DO DÉFICIT DE ATENÇÃO/HIPERATIVIDADE
}

\author{
Marcia Cavadas', Liliane Desgualdo Pereira², Paulo Mattos³
}

\begin{abstract}
RESUMO - Objetivo: Comparar o desempenho em teste de avaliação do processamento auditivo (PA) num grupo de crianças e adolescentes com transtorno do déficit de atenção e hiperatividade (TDAH) pré e pósmetilfenidato. Método: Foram aplicados testes comportamentais padronizados de avaliação do PA em 29 indivíduos com TDAH na faixa etária de 7 a 15 anos, de ambos os sexos. Utilizou-se um grupo de comparação de 29 indivíduos sem déficit de atenção, com e sem transtornos de aprendizado. Resultados: 0 grupo com TDAH apresentou desempenho nos testes de PA semelhante ao grupo controle sem transtornos do aprendizado sendo seus resultados ainda melhores na ocasião pós-medicação. O grupo sem déficit de atenção e com problemas de aprendizagem apresentou o pior desempenho nos testes de PA e o grupo sem déficit de atenção e sem problemas de aprendizagem foi o que apresentou o melhor desempenho. Conclusão: O teste de PA não permitiu diferenciar portadores de TDAH de controles pareados; o metilfenidato se associou a melhora do desempenho nos testes de PA nos portadores de TDAH.
\end{abstract}

PALAVRAS-CHAVE: percepção auditiva, atenção, transtorno do déficit de atenção, TDAH.

\section{Effects of methylphenidate in auditory processing evaluation of children and adolescents with attention deficit hyperactivity disorder}

\begin{abstract}
Purpose: To compare the performance of a group of children and adolescents diagnosed with attention deficit hyperactivity disorder (ADHD) pre and post-methylphenidate use in a behavioral auditory processing test battery (AP). Method: Twenty-nine subjects, male and female, ranging from 7 to 15 years old have undergone different behavioral auditory processing tests. A control group composed of 29 subjects with and without learning disabilities was also evaluated. Results: The group with ADHD had a performance similar to the control group without learning disabilities which improved after medication. The group with learning disabilities and without ADHD had the worst performance in tests while the group without learning disabilities and without ADHD exhibited the best ones. Conclusion: The AP battery was unable to distinguish ADHD patients from paired controls; the use of methylphenidate improved the performance on AP tests of ADHD group in the post-medication evaluation.
\end{abstract}

KEY WORDS: auditory perception attention, attention-deficit disorder, ADHD.

O transtorno do déficit de atenção/hiperatividade (TDAH) é caracterizado por dificuldades de modulação da atenção e/ou hiperatividade/impulsividade. É um transtorno muito comum na infância $(5,8 \%$ das crianças, aproximadamente, em nosso meio, utilizando os critérios do sistema Diagnostic and Statistical Manual - DSM-IV, da Associação Americana de Psiquiatria $\left.{ }^{1}\right)$, comprometendo o desempenho escolar, dificultando as relações interpessoais e provocando baixa auto-estima. O diagnóstico de TDAH é realizado com informações colhidas junto aos pais e professores e também por meio de observação clínica da criança. Os sintomas devem estar presentes por, pelo menos, seis meses, comprometer o funcionamento acadêmico ou social e ter início antes dos sete anos de idade. De acordo com o DSM IV, o diagnóstico exige pelo menos

Programa de Pós-Graduação em Distúrbios da Comunicação Humana - Campo Fonoaudiológico - Departamento de Fonoaudiologia da Universidade Federal de São Paulo, São Paulo SP - Brasil (UNIFESP) e Grupo de Estudos do Déficit de Atenção (GEDA) da Universidade Federal do Rio de Janeiro, Rio de Janeiro RJ - Brasil (UFRJ): ${ }^{1}$ Fonoaudióloga, Professora Doutora do Curso de Fonoaudiologia da Faculdade de Medicina da UFRJ; ${ }^{2}$ Fonoaudióloga, Professora Doutora do Departamento de Fonoaudiologia da UNIFESP; ${ }^{3}$ Psiquiatra, Professor Doutor da Faculdade de Medicina da UFRJ.

Recebido 26 Maio 2006, recebido na forma final 4 Setembro 2006. Aceito 25 Outubro 2006. 
seis sintomas no âmbito da desatenção e/ou da hiperatividade/impulsividade. Quando o diagnóstico é realizado precocemente, menores serão as conseqüências psicológicas na criança ao longo dos anos. O tratamento mais utilizado para o TDAH é a farmacoterapia ${ }^{2}$. Não há evidências até o momento de benefícios proporcionados pela psicoterapia, ao menos para o controle dos sintomas primários.

O processamento auditivo (PA) constitui uma série de processos envolvidos nas habilidades auditivas, incluindo atenção, memória, detecção do som, localização, figura-fundo, entre outras. A avaliação do PA, com testes comportamentais padronizados, tem sido realizada aqui no Brasil desde 1993, contribuindo de maneira significativa na terapêutica fonoaudiológica ${ }^{3}$. Observando-se a correlação da desatenção de crianças com desordem do PA e sabendo-se que o TDAH é um distúrbio de atenção, Vilanova ${ }^{4}$ caracterizou o TDAH por meio de manifestações clínicas e avaliou o PA de um grupo de crianças. $O$ autor encontrou evidências de alteração do PA em indivíduos portadores dessa síndrome, através do teste dicótico verbal, com estímulo consoante-vogal. Outros autores estudaram a correlação entre PA e TDAH ${ }^{5-10}$. No entanto, o efeito do uso de medicamento no desempenho em testes de PA ainda não foi estudado. Este estudo tem como objetivo comparar o desempenho em teste de avaliação de PA num grupo de crianças e adolescentes com TDAH pré e pós-tratamento com metilfenidato.

\section{MÉTODO}

A pesquisa foi realizada no Ambulatório de Audiologia Clínica do Curso de Fonoaudiologia da Faculdade de Medicina da Universidade Federal do Rio de Janeiro (UFRJ). Este estudo é parte do projeto de pesquisa multidisciplinar, de que participam psiquiatras, neurologistas, psicólogos e fonoaudiólogos, denominado Grupo de Estudos sobre Déficit de Atenção (GEDA) / UFRJ. O projeto engloba, além da avaliação do comportamento auditivo, avaliação de linguagem, avaliação neurológica, psiquiátrica e uma neuropsicológica, tendo sido aprovado pelo Comitê de Ética em Pesquisa do Instituto de Psiquiatria da UFRJ.

Na primeira consulta, orientava-se o indivíduo quanto às demais avaliações que ele participaria e era assinado o termo de consentimento livre e esclarecido. Foram entrevistados de modo consecutivo crianças e adolescentes autoreferidos ou encaminhados de outros serviços ao GEDA. Uma triagem inicial compreendia rastreio com especialista com experiência em TDAH, sendo os casos suspeitos com base no questionário SNAP-IV encaminhados para uma entrevista de base, quando era aplicada a entrevista semi-estruturada P-Chips (Children's Interview for Psychiatric Syndromes) que utiliza os critérios diagnósticos da DSM-IV. Na mesma entrevista eram investigadas eventuais comorbidades (transtornos do humor, transtornos de ansiedade, transtornos disruptivos, transtorno de oposição e desafio e transtorno de conduta).

Após a confirmação do diagnóstico de TDAH e a determinação dos subtipos (predominantemente desatento; predominantemente hiperativo-impulsivo e combinado) o paciente era submetido a avaliação de QI estimado, utilizando os subtestes blocos e vocabulário da bateria Wechsler de inteligência (WISC-III) ${ }^{11}$.

A avaliação do PA ocorreu antes do início do tratamento medicamentoso prescrito pelo médico psiquiatra. A rotina do atendimento médico era de acompanhamento quinzenal.

Como critérios de inclusão, no grupo com déficit de atenção, os indivíduos deveriam apresentar diagnóstico de TDAH, apresentar sensibilidade auditiva dentro dos padrões de normalidade e não ser portador de enfermidade otológica. Os critérios de exclusão nesse grupo foram: QI inferior a 80 , presença de transtornos de aprendizagem e de comunicação, presença de doença neurológica, transtornos abrangentes do desenvolvimento e psicoses.

Foram incluídos de modo consecutivo 38 indivíduos de ambos os sexos na faixa etária de 7 a 15 anos, 29 destes retornando para reavaliação, após o tratamento medicamentoso. Este grupo foi denominado de grupo com déficit de atenção (GDA) (Tabela 1). Esses indivíduos foram recrutados e atendidos no GEDA / UFRJ.

O grupo de comparação foi formado por uma amostra de conveniência pareada por sexo e faixa etária. Nesse grupo foram incluídos indivíduos que não apresentavam diagnóstico de TDAH com ou sem transtornos de aprendizagem e de comunicação e/ou alterações auditivas. Sendo assim, foram selecionados 29 indivíduos para constituir o grupo de comparação denominado de grupo sem déficit de atenção - GS. O grupo de comparação foi subdividido separando-se as variáveis com e sem transtornos de aprendizagem e de comunicação e/ou alterações auditivas. $O$ grupo sem déficit de atenção e com possíveis alterações de aprendizagem e de comunicação e/ou auditivas foi denominado GS1 (Tabela 1). O grupo sem déficit de atenção e sem possíveis alterações de aprendizagem e de comunicação e/ou alterações auditivas foi denominado GS2 (Tabela 1). Utilizaram-se como critérios de elegibilidade para o grupo GS2 os indivíduos cujos resultados do índice percentual de reconhecimento de fala (IPRF) fossem maiores que ou iguais a $88 \%$, avaliação simplificada do PA sem alteração e ausência de evidências de problemas de aprendizagem e comunicação detectados por anamnese realizada com os pais e/ou responsáveis.

O equipamento utilizado para a realização das avaliações auditivas foi o audiômetro da marca Interacoustic, modelo CE 10, com fone TDH - 39 e coxim MX-41, calibrado segundo o padrão ANSI - 69 acoplado a um CD player. Os estímulos utilizados para avaliação de PA foram os gravados nos CDs volumes 1 e 2 do manual de avaliação de processamento auditivo central ${ }^{3}$. Os exames foram realizados em cabina acústica cujos níveis de pressão sonora em cada freqüência estavam de acordo com o nível máximo permissível de ruído ambiental, obedecendo, assim, à norma ANSI S31991. Ainda as medidas de imitância acústica foram obtidas com o imitanciômetro modelo Az 7 da marca Interacoustic. 
Inicialmente, os indivíduos selecionados tiveram seus pais e/ou responsáveis submetidos a anamnese com questões relativas ao desenvolvimento de fala, audição e linguagem, dificuldades na alfabetização, rendimento escolar e características comportamentais de tais indivíduos.

Com o objetivo de determinar o limiar mínimo de audibilidade e descartar enfermidades otológicas, foi realizada avaliação audiológica básica. Essa avaliação constou de audiometria tonal liminar por via aérea, detecção do limiar de reconhecimento de fala (SRT - Speech Reception Treshold) e medidas de imitância acústica. A imitanciometria foi realizada com objetivo de descartar possíveis alterações de ouvido médio, excluindo desta pesquisa, indivíduos com otites e disfunção da tuba auditiva. Em seguida os indivíduos foram submetidos a avaliação das habilidades auditivas por meio de testes especiais comportamentais, que para fins de facilitar a explanação foram divididos em dois grupos: avaliação simplificada do PA, abreviada neste estudo como "ASPA", e testes especiais comportamentais, abreviados como "TEC".

A ASPA incluiu: teste de localização sonora (LS) ${ }^{13}$, teste de memória seqüencial para sons verbais (MSV) ${ }^{13,14}$; teste de memória seqüencial para sons não verbais (MSNV) ${ }^{13}$.
Diversos processos acústicos contribuem para que seja possível reconhecer sons incompletos, incluindo o fechamento e a atenção seletiva. No teste de fala com ruído branco $(F / R)^{15}$, faz parte da interpretação comparar os acertos obtidos na presença de ruído com aqueles obtidos na ausência de ruído. Sendo assim, foi estudada também a pesquisa do IPRF com gravação, que utiliza a mesma lista de palavras do teste de F/R, em outra ordem de apresentação. Para a realização destes testes (F/R e IPRF) foi utilizado o CD número 1, faixa $2^{3}$. O teste dicótico de dissílabos alternados SSW (Staggered Spondaic Word Test) foi desenvolvido nos Estados Unidos por Katz, em 1962, e sua versão em português foi proposta por Borges, Rejtman e Schneider supervisionada pelo próprio autor do teste ${ }^{16}$. Para a realização deste teste foi utilizado o $C D$ número 2 , faixa $6^{3}$. Os testes F/R, IPRF e SSW foram denominados, neste estudo, como TEC.

A avaliação do PA nos indivíduos do grupo GDA foi realizada em dois momentos: pré e pós-administração do metilfenidato, um psicoestimulante, em doses variáveis de acordo com a tolerabilidade e a eficácia clínica, respeitando os limites de 0,5 a 0,8 mg/kg, de acordo com a literatura ${ }^{12}$. O intervalo entre a primeira avaliação, sem a medicação,

Tabela 1. Distribuição dos indivíduos dos grupos GDA, GS1 e GS2 segundo a faixa etária e escolaridade.

\begin{tabular}{|c|c|c|c|c|c|c|}
\hline $\begin{array}{c}\text { Indivíduos } \\
\text { GDA }\end{array}$ & $\begin{array}{c}\text { Faixa etária } \\
\text { em anos }\end{array}$ & $\begin{array}{c}\text { Tempo de } \\
\text { escolaridade } \\
\text { em anos }\end{array}$ & & $\begin{array}{l}\text { Indivíduos } \\
\text { GS1 e GS2 }\end{array}$ & $\begin{array}{c}\text { Faixa etária } \\
\text { em anos }\end{array}$ & $\begin{array}{c}\text { Tempo de } \\
\text { escolaridade } \\
\text { em anos }\end{array}$ \\
\hline 1 & 7 & 1 & GS1 & 1 & 7 & 1 \\
\hline 2 & 7 & 1 & & 2 & 7 & 1 \\
\hline 3 & 8 & 2 & & 3 & 8 & 1 \\
\hline 4 & 8 & 1 & & 4 & 8 & 2 \\
\hline 5 & 8 & 2 & & 5 & 8 & 2 \\
\hline 6 & 8 & 2 & & 6 & 8 & 2 \\
\hline 7 & 8 & 3 & & 7 & 8 & 2 \\
\hline 8 & 8 & 3 & & 8 & 8 & 3 \\
\hline 9 & 9 & 2 & & 9 & 9 & 2 \\
\hline 10 & 9 & 2 & & 10 & 9 & 3 \\
\hline 11 & 9 & 2 & & 11 & 9 & 3 \\
\hline 12 & 9 & 3 & & 12 & 9 & 3 \\
\hline 13 & 9 & 4 & & 13 & 9 & 4 \\
\hline 14 & 10 & 4 & & 14 & 10 & 3 \\
\hline 15 & 10 & 5 & & 15 & 10 & 4 \\
\hline 16 & 10 & 4 & & 17 & 10 & 4 \\
\hline 17 & 10 & 3 & & 20 & 11 & 5 \\
\hline 18 & 10 & 3 & & 22 & 11 & 5 \\
\hline 19 & 11 & 6 & & 25 & 12 & 6 \\
\hline 20 & 11 & 5 & GS2 & 16 & 10 & 4 \\
\hline 21 & 11 & 5 & & 18 & 10 & 5 \\
\hline 22 & 11 & 4 & & 19 & 11 & 4 \\
\hline 23 & 12 & 4 & & 21 & 11 & 5 \\
\hline 24 & 12 & 7 & & 23 & 12 & 5 \\
\hline 25 & 12 & 5 & & 24 & 12 & 6 \\
\hline 26 & 13 & 7 & & 26 & 13 & 7 \\
\hline 27 & 14 & 6 & & 27 & 15 & 8 \\
\hline 28 & 15 & 9 & & 28 & 15 & 9 \\
\hline 29 & 15 & 8 & & 29 & 15 & 9 \\
\hline Média & 10,14 & 3,9 & & Média & 10,17 & 4,07 \\
\hline Desvio padrão & 2,22 & 2,13 & & Desvio padrão & 2,29 & 2,24 \\
\hline Mínimo & $\begin{array}{c}2,22 \\
7\end{array}$ & 1 & & Mínimo & 7 & 1 \\
\hline Máximo & 15 & 9 & & Máximo & 15 & 9 \\
\hline
\end{tabular}

GDA, grupo com déficit de atenção; GS1, grupo sem déficit de atenção e com possíveis alterações de aprendizagem e de comunicação e/ou auditivas; GS2, grupo sem déficit de atenção e sem possíveis alterações de aprendizagem e de comunicação e/ou alterações auditivas. 
e a segunda avaliação, com a medicação, variou entre 2 e 9 meses. O momento da reavaliação após o uso contínuo do medicamento foi selecionado tendo como critério o relato pelos familiares ao médico do GEDA de remissão dos sintomas de TDAH.

A avaliação do PA nos indivíduos do grupo GS também foi realizada em dois momentos, mantendo um intervalo mínimo de 15 dias entre eles.

No projeto GEDA, cada indivíduo diagnosticado com TDAH poderia participar de uma sessão de avaliação de PA. Sendo assim, foi selecionado apenas o teste SSW para ser realizado duas vezes numa mesma sessão e verificar o efeito dessa repetição no desempenho do indivíduo, independentemente da ocasião. Neste estudo foram utilizados os termos SSW-teste e SSW-reteste para designar a primeira e a segunda avaliação numa mesma sessão de avaliação do PA. Foi feita uma análise comparativa entre os grupos estudados: GDA, GS1 e GS2.

Para estudar as diferenças entre os grupos GDA, GS1 e GS2, entre as ocasiões pré e pós e a interação entre grupo e ocasião foi realizada análise de variância com medidas repetidas dos testes memória seqüencial verbal, memória seqüencial não-verbal e as condições não competitivas, competitivas de ambas orelhas e total de acertos no SSW-teste e no SSW-reteste. O nível de significância adotado foi de 0,05 ou $5 \%$ e assinalaram-se com asterisco os valores significativos.

\section{RESULTADOS}

$\mathrm{Na}$ avaliação simplificada do PA (ASPA) os resulta- dos dos testes apresentaram-se normais em todos os grupos estudados. O grupo GS2 apresentou os meIhores resultados, seguido dos grupos GDA e do GS1. $\mathrm{Na}$ Tabela 2 apresentam-se as médias e os desvios padrão dos testes da ASPA (MSV, MSNV e LS) nos grupos GDA, GS1 e GS2 nas duas ocasiões pré e pós. Para os testes especiais comportamentais (TEC) (Tabelas 2 e 3), o grupo GS2 apresentou o melhor desempenho, em média. Nos testes IPRF e F/R o grupo GS1 apresentou o pior desempenho, em média, e no SSW, independentemente da etapa SSW-teste ou SSW-reteste, os resultados dos grupos GS1 e GDA foram mais semelhantes e inferiores do que os resultados do GS2. Para as condições não competitivas (DNC e ENC) no SSW-reteste, não foram detectadas diferenças entre as médias dos três grupos, tanto na ocasião pré quanto na pós. De modo geral, em todos os procedimentos realizados neste estudo, as médias observadas na ocasião pós foram maiores (melhores) do que na ocasião pré, e a variabilidade dos resultados da ocasião pós foi menor do que a variabilidade dos resultados da ocasião pré. Na Tabela 3 mostram-se as médias e os desvios padrão do TEC (IPRF e F/R) nos grupos GDA, GS1 e GS2 nas duas ocasiões pré e pós.

$\mathrm{Na}$ análise de variância com medidas repetidas nos testes de MSV, MSNV e no SSW em todas as condições - DNC, ENC, DC, EC e total - (Tabela 4) houve resul-

Tabela 2. Desempenho nos testes de MSV, MSNV e LS nos grupos GDA, GS1 E GS2 nas ocasiões pré e pós metilfenidato.

\begin{tabular}{|c|c|c|c|c|c|c|c|}
\hline \multirow{2}{*}{$\begin{array}{l}\text { Grupos } \\
N\end{array}$} & & \multicolumn{2}{|c|}{ MSV } & \multicolumn{2}{|c|}{ MSNV } & \multicolumn{2}{|c|}{ Localização sonora } \\
\hline & & pré & pós & pré & pós & pré & pós \\
\hline GDA & Média & 2,24 & 2,38 & 2,55 & 2,72 & 4,72 & 4,97 \\
\hline $\mathrm{N}=29$ & Desvio padrão & 0,95 & 0,73 & 0,63 & 0,45 & 0,59 & 0,19 \\
\hline GS1 & Média & 1,84 & 2,16 & 2,32 & 2,16 & 5,0 & 5,0 \\
\hline$N=19$ & Desvio padrão & 0,83 & 0,83 & 0,60 & 0,60 & 0,0 & 0,0 \\
\hline GS2 & Média & 2,30 & 2,70 & 2,90 & 2,80 & 4,90 & 5,0 \\
\hline$N=10$ & Desvio padrão & 0,48 & 0,48 & 0,32 & 0,42 & 0,32 & 0,0 \\
\hline
\end{tabular}

MSV, memória seqüencial verbal; MSNV, memória seqüencial não-verbal; LS, localização sonora.

Tabela 3. Desempenho nos testes IPRF e FIR por OD e OE, nas duas ocasiões, pré e pós metilfenidato, nos grupos GDA, GS1 E GS2.

\begin{tabular}{|c|c|c|c|c|c|c|c|c|c|}
\hline \multirow{3}{*}{$\begin{array}{l}\text { Grupos } \\
\mathrm{N}\end{array}$} & & \multicolumn{4}{|c|}{ IPRF } & \multicolumn{4}{|c|}{ F/R } \\
\hline & & \multicolumn{2}{|c|}{ OD } & \multicolumn{2}{|c|}{$\mathrm{OE}$} & \multicolumn{2}{|c|}{ OD } & \multicolumn{2}{|c|}{$\mathrm{OE}$} \\
\hline & & pré & pós & pré & pós & pré & pós & pré & pós \\
\hline GDA & Média & 86,21 & 89,79 & 88,55 & 90,90 & 76,15 & 83,03 & 74,07 & 81,38 \\
\hline$N=29$ & Desvio padrão & 7,06 & 3,79 & 5,73 & 4,26 & 11,61 & 6,98 & 12,27 & 8,23 \\
\hline GS1 & Média & 83,16 & 87,58 & 87,37 & 90,32 & 67,37 & 73,68 & 62,95 & 75,16 \\
\hline$N=19$ & Desvio padrão & 5,26 & 4,60 & 6,29 & 6,01 & 6,70 & 8,57 & 6,51 & 8,59 \\
\hline GS2 & Média & 92,00 & 93,60 & 92,00 & 94,40 & 78,40 & 88,40 & 78,40 & 88,40 \\
\hline$N=10$ & Desvio padrão & 2,67 & 4,30 & 2,67 & 2,80 & 5,71 & 4,79 & 5,72 & 4,79 \\
\hline
\end{tabular}

IPRF, índice percentual de reconhecimento de fala; F/R, fala com ruído; OD, orelha direita; OE, orelha esquerda. 
Tabela 4. Acertos por condição DNC, DC, EC, ENC e total de acertos para o SSW-teste e para o SSW-reteste, por ocasião pré e pós metilfenidato, nos grupos GDA, GS1 e GS2.

\begin{tabular}{|c|c|c|c|c|c|c|c|c|}
\hline & & & \multicolumn{2}{|c|}{ GDA } & \multicolumn{2}{|c|}{ GS1 } & \multicolumn{2}{|c|}{ GS2 } \\
\hline & & & Média & $\begin{array}{l}\text { Desvio } \\
\text { padrão }\end{array}$ & Média & $\begin{array}{l}\text { Desvio } \\
\text { padrão }\end{array}$ & Média & $\begin{array}{l}\text { Desvio } \\
\text { padrão }\end{array}$ \\
\hline \multirow[t]{10}{*}{ SSW-teste } & DNC & pré & 93,53 & 6,07 & 91,18 & 5,49 & 96,75 & 3,55 \\
\hline & & pós & 96,55 & 3,92 & 95,79 & 3,91 & 99,25 & 1,69 \\
\hline & $\mathrm{DC}$ & pré & 81,55 & 12,84 & 79,08 & 10,42 & 90,75 & 7,08 \\
\hline & & pós & 87,76 & 11,94 & 87,89 & 5,35 & 96,85 & 2,85 \\
\hline & $\mathrm{EC}$ & pré & 73,40 & 19,14 & 74,87 & 11,80 & 91,50 & 5,03 \\
\hline & & pós & 84,66 & 15,89 & 86,18 & 8,75 & 95,75 & 2,65 \\
\hline & ENC & pré & 94,72 & 6,79 & 91,71 & 6,77 & 98,50 & 2,10 \\
\hline & & pós & 96,72 & 4,39 & 96,18 & 4,36 & 98,50 & 2,10 \\
\hline & Total & pré & 85,66 & 9,75 & 84,21 & 7,47 & 94,38 & 3,91 \\
\hline & & pós & 91,42 & 7,83 & 91,51 & 4,58 & 97,59 & 1,93 \\
\hline \multirow[t]{10}{*}{ SSW-reteste } & DNC & pré & 95,52 & 5,61 & 93,81 & 5,67 & 98,75 & 1,78 \\
\hline & & pós & 97,33 & 3,89 & 96,32 & 4,74 & 99,00 & 1,75 \\
\hline & DC & pré & 86,55 & 11,43 & 85,13 & 6,79 & 94,5 & 4,37 \\
\hline & & pós & 91,03 & 9,17 & 89,21 & 5,95 & 97,25 & 3,80 \\
\hline & $\mathrm{EC}$ & pré & 80,00 & 16,97 & 80,92 & 10,55 & 95,00 & 3,73 \\
\hline & & pós & 88,17 & 12,40 & 88,03 & 7,66 & 97,00 & 3,07 \\
\hline & ENC & pré & 96,90 & 4,31 & 94,34 & 7,49 & 98,75 & 1,77 \\
\hline & & pós & 98,36 & 2,70 & 96,45 & 4,88 & 99,00 & 1,75 \\
\hline & Total & pré & 89,74 & 8,51 & 88,55 & 6,33 & 96,75 & 2,16 \\
\hline & & pós & 91,42 & 7,83 & 91,51 & 4,58 & 97,59 & 1,94 \\
\hline
\end{tabular}

DNC, direita não competitiva; DC, direita competitiva; EC, esquerda competitiva; ENC, esquerda não competitiva.

Tabela 5. Níveis descritivos (p-valores) obtidos na análise de variância para os testes MSV e MSNV e para as condições $D N C, D C, E C, E N C$ e do total de acertos para o SSW-teste e para o SSW-reteste por grupo e por ocasião.

\begin{tabular}{|c|c|c|c|c|}
\hline & \multirow[t]{2}{*}{ Testes } & \multicolumn{3}{|c|}{$\mathrm{p}$-valor } \\
\hline & & Grupo & Ocasião & $\begin{array}{c}\text { Interação } \\
\text { grupo x ocasião }\end{array}$ \\
\hline & MSV & $0,123^{*}$ & $0,028^{*}$ & 0,648 \\
\hline & MSNV & $0,004^{*}$ & 0,781 & 0,252 \\
\hline \multirow[t]{5}{*}{ SSW-teste } & DNC & $0,029 *$ & $0,000 *$ & 0,331 \\
\hline & DC & $0,023^{*}$ & $0,000 *$ & 0,318 \\
\hline & $\mathrm{EC}$ & $0,014^{*}$ & $0,000 *$ & 0,070 \\
\hline & ENC & $0,044^{*}$ & $0,004^{*}$ & 0,119 \\
\hline & Total & $0,010^{*}$ & $0,000 *$ & 0,062 \\
\hline \multirow[t]{5}{*}{ SSW-reteste } & DNC & 0,083 & $0,004^{*}$ & 0,262 \\
\hline & DC & $0,022^{*}$ & $0,000 *$ & 0,679 \\
\hline & EC & $0,017^{*}$ & 0,000 * & 0,053 \\
\hline & ENC & 0,073 & $0,012^{*}$ & 0,386 \\
\hline & Total & $0,016^{*}$ & $0,000 *$ & 0,091 \\
\hline
\end{tabular}

MSV, memória seqüencial verbal; MSNV, memória seqüencial não-verbal; DNC, direita não competitiva; DC, direita competitiva; EC, esquerda competitiva; ENC, esquerda não competitiva. (*)p<0,05. 
tados significativos ( $p$-valor abaixo de 0,05 ) em quase todos os testes desta análise para a comparação entre grupos e ocasião. Não houve interação entre grupo e ocasião. Na Tabela 4 mostram-se as médias e desvios padrão do TEC (SSW-teste e SSW-reteste) nos grupos GDA, GS1 e GS2 nas duas ocasiões pré e pós.

$\mathrm{Na}$ Tabela 5 demonstram-se os níveis descritivos ( $p$-valores) obtidos na análise de variância nos testes MSV, MSNV, nas condições competitivas, não competitivas e no total de acertos para o SSW-teste e para o SSW-reteste por grupo e por ocasião.

\section{DISCUSSÃO}

O diagnóstico de TDAH permanece sendo clínico, inexistindo exames complementares para o mesmo até o momento. Alguns exames parecem estar indicados em casos específicos, quando existe necessidade de se investigar a presença de comorbidade ou há necessidade de se esclarecer um diagnóstico diferencial em particular. O relato de um caso diagnosticado e tratado como TDAH desde a infância, sob uso de metilfenidato desde os 9 anos de idade, apresentando DPA não indentificado previamente foi relatado ${ }^{8}$, ilustra tais casos.

Alguns autores observaram diferenças significativas no TDAH com relação aos limiares de fusão auditiva, com limiares mais pobres, piores, do que as crianças com problemas de linguagem e crianças normais ${ }^{9}$. Um estudo encontrou evidências de alteração do PA em indivíduos portadores de TDAH4. Em estudo realizado com indivíduos portadores de TDAH medicados e não medicados não foi observada diferença significativa no desempenho dos testes de $\mathrm{PA}^{6}$. Na avaliação de potenciais auditivos em crianças com TDAH houve diferença entre os resultados dos indivíduos medicados em comparação com os não medicados ${ }^{10}$. No presente estudo, embora tenham sido observadas diferenças qualitativas no desempenho dos testes de PA na ocasião pós-medicação, o grupo de portadores de TDAH apresentou bom desempenho na avaliação de PA demonstrando uma tendência de ausência de alteração nos testes de PA. O uso do metilfenidato se associou a melhora do desempenho nos portadores de TDAH.

Em conclusão, crianças e adolescentes portadores de TDAH sem evidências de transtornos de aprendizado não apresentaram desempenho comprometido em tarefas de PA, embora o mesmo seja algo inferior ao observado em uma amostra comparativa sem TDAH numa avaliação qualitativa. As diferenças observadas, entretanto, não permitem estabelecer aquelas tarefas como método auxiliar de diagnóstico dife- rencial. Crianças e adolescentes portadores de TDAH submetidos a tratamento farmacológico com metilfenidato em doses terapêuticas apresentam incrementos no seu desempenho em tarefas de PA, o que parece ser atribuível à melhora nos níveis de atenção durante as tarefas propostas. O grupo de portadores de TDAH, como um todo, apresentou menor variabilidade das respostas pós-medicamento, embora o significado clínico deste achado não seja claro. $O$ desenho deste estudo, entretanto, não permite estabelecer a relação entre o uso do medicamento e a meIhora no desempenho. Um desempenho comprometido em tarefas de PA no estudo em questão esteve mais associado à presença de transtornos de aprendizado e comunicação, corroborando a hipótese que as dificuldades no processamento eventualmente observadas em portadores de TDAH não representam um déficit primário, sendo mais bem entendidas como um fenômeno secundário à desatenção.

\section{REFERÊNCIAS}

1. Rohde LA, Szobot C, Polanczyk G, Schmitz M, Martins S, Tramontina S. Attention deficit-hiperactivity disorder in a diverse culture: do research and clinical findings support the notion of a cultural construct for the disorder ? Biol Psychiatry 2005;57:1436-1441.

2. Correia AG Filho, Pastura G. As medicações estimulantes. In Rohde LA, Mattos P (Eds). Princípios e práticas em transtorno do déficit de atenção/hiperatividade. Porto Alegre: Artmed, 2003:161-173.

3. Pereira LD, Schochat E. Processamento auditivo central: manual de avaliação. São Paulo: Lovise, 1997.

4. Vilanova LCP. Distúrbios de atenção na infância e adolescência. Rev Neuro 1993;2:30-32.

5. Love AJ, Thompson NGG. Language disorders and attention deficit disorders in young children referred for psychiatric services: analysis of prevalence and a conceptual synthesis. Am J Orthopsychiatry 1988; 52:52-64.

6. Tillery KL. Central auditory processing abilities of attention deficit hiparactivity disordered children: with and without methylphenidate [Tese]. Illinos, 1992.

7. Bellis TJ, Ferre JM. Multidimensional approach to the differential diagnosis of central auditory processing disorders in children. J Am Acad Audiol 1999;10:319-328.

8. Sanchez ML, Misorelli MIL, Alvarez AMMA. Benefícios do esclarecimento da comorbidade de transtorno de déficit de atenção/hiperatividade (TDAH) e distúrbio do processamento auditivo central (CAPD): estudo de caso. Rev Bras Med Otorrinolaringol 1999;6:62-64.

9. Feniman MR, Keith RW, Cunningham RF. Assessment of auditory processing in children with attention deficit hyperactivity disorder and language-based learning impairments. Disturb Comum 1999;11:9-27.

10. Schochat E, Scheuer CI, Andrade ER. ABR and auditory P300 findings in children with ADHD. Arq Neuropsiquiatr 2002;60:742-747.

11. Wechsler D Wisc-III Manual the psychological corporation. New York: Psychological Corporation, 1991.

12. Pastura G, Mattos P. Efeitos colaterais do metilfenidato. Rev Psiq Clin 2004;31:100-104.

13. Pereira LD. Processamento auditivo central: uma abordagem passo a passo. In Pereira LD, Schochat E (Eds). Processamento auditivo central: manual de avaliação. São Paulo: Lovise, 1997:49-59.

14. Corona AP. Processamento auditivo: seqüencialização verbal de três e quatro sílabas em escolares de 3 a 12 anos [Monografia]. Salvador: Universidade Federal da Bahia, 2000.

15. Schochat E, Pereira LD. Fala com ruído. In Pereira LD, Schochat E (Eds). Processamento auditivo central: manual de avaliação. São Paulo: Lovise, 1997:99-102.

16. Borges ACLC. Adaptação do teste SSW para a língua portuguesa nota preliminar. Acta AWHO 1986;5(Suppl1):S38-S40. 